\title{
Impact of an Exercise Training Program in a Patient with Relapsing Polychondritis: A Case Report
}

\author{
Alexandre Moura dos Santos, Diego Sales de Oliveira, Rafael Giovani Misse, \\ Letícia Alves Perin, Jean Marcos de Souza, Fernanda Rodrigues Lima, \\ Rosa Maria Rodrigues Pereira, Samuel Katsuyuki Shinjo*
}

Division of Rheumatology, Hospital das Clinicas HCFMUSP, Faculdade de Medicina, Universidade de Sao Paulo, Sao Paulo, Brazil

Email: *samuel.shinjo@gmail.com

How to cite this paper: dos Santos, A.M., de Oliveira, D.S., Misse, R.G., Perin, L.A., de Souza, J.M., Lima, F.R., Pereira, R.M.R. and Shinjo, S.K. (2018) Impact of an Exercise Training Program in a Patient with Relapsing Polychondritis: A Case Report. Open Journal of Rheumatology and Autoimmune Diseases, 8, 93-98.

https://doi.org/10.4236/ojra.2018.83010

Received: August 3, 2018

Accepted: August 26, 2018

Published: August 29, 2018

Copyright $\odot 2018$ by authors and Scientific Research Publishing Inc. This work is licensed under the Creative Commons Attribution International License (CC BY 4.0).

http://creativecommons.org/licenses/by/4.0/

\begin{abstract}
Background: Exercise training has demonstrating to be safe and promote benefits for several rheumatologic autoimmune diseases. However, no study has evaluated the safety and benefits of exercise in relapsing polychondritis. Aim: To evaluate the effectiveness of an exercise training program in a patient with relapsing polychondritis. Case presentation: A 67-year-old female patient with relapsing polychondritis in remission was submitted to a 12 -week, twice weekly, aerobic and resistance training program. Aerobic capacity, muscle strength and function capacity, as well as body composition, were evaluated at baseline and after 12-weeks. Conclusions: Exercise training program demonstrated to be effective for increasing aerobic capacity, muscle strength and function, and for improving body composition in the patient. Further studies are necessary to confirm these findings.
\end{abstract}

\section{Keywords}

Exercise Training, Relapsing Polychondritis, Aerobic Training, Resistance Training

\section{Introduction}

Relapsing polychondritis (RP) is a rare autoimmune systemic disease characterized by inflammation of proteoglycan-rich structures and cartilaginous tissues [1] [2]. Patients with RP have recurrent inflammation of auricular pinna, nasal cartilage, peripheral joints and vertebral disks [2] [3]. Moreover, these patients 
may exhibit clinical manifestations, such as auricular chondritis, non-erosive polyarthritis, nasal cartilage chondritis, inflammation of ocular structures, respiratory tract chondritis, as well as vestibular and cochlear damage [2] [3] [4].

Structural deformities can lead to life-threatening cardiopulmonary manifestations, such as airway collapse and valvarcardiac regurgitation [4] [5]. Generally, the primary cause of death is respiratory failure due to RP, followed by lung infections and cardiovascular events [1] [2] [3] [4] [5].

Exercise training has proven to be an important tool for mitigating clinically related symptoms in autoimmune rheumatic diseases, such as systemic lupus erythematosus, rheumatoid arthritis and systemic autoimmune myopathies [6] [7]. In addition, exercise training has been shown to promote an increase in aerobic capacity, muscle strength and function, and to improve quality of life in autoimmune rheumatic diseases [6] [7] [8] [9]. Importantly, these benefits of exercise training have been observed without exacerbating the inflammatory milieu [9] [10], proving that this non-pharmacological strategy is effective and safe for the management of autoimmune rheumatic diseases. However, to the best of our knowledge, there are no studies evaluating the effectiveness and safety of exercise training programs for patients with RP.

\section{Case Study}

A 67-year-old female with RP, according to criteria of McAdam et al. [2] was assessed in the present case study. She presented the first symptoms 10 years prior, with polyarthralgia, bilateral diffuse anterior scleritis, hoarseness and acute chondritis of the right auricle. There was no cardiac or interstitial lung involvement. At the time of the initial manifestations, she was an active attorney, non-smoker, with controlled diabetes mellitus and systemic arterial hypertension. She was promptly treated with the combination of methotrexate (15 $\mathrm{mg} /$ week) and prednisone $(0.5 \mathrm{mg} / \mathrm{kg} /$ day $)$, with complete resolution of symptoms. One year later, she presented hoarseness and ear chondritis again, during glucocorticoid withdrawal, and was successfully treated with a new prednisone dose $(0.5 \mathrm{mg} / \mathrm{kg} /$ day again) and methotrexate adjustment (to $25 \mathrm{mg} /$ week). Her disease has since remained stable, without related complaints and normal laboratory tests. Prednisone was suspended 8 years ago and methotrexate 2 years later, without further relapse.

The patient underwent a 12-week, twice weekly, supervised exercise training program at an intra-hospital gymnasium. The exercise training program was comprised of aerobic and resistance training. Aerobic sessions consisted of a 5-min warm-up followed by 30 - 50 min of treadmill walking, and a 5-min cooling-down period. Walking time was gradually increased every four weeks, from 30 to $50 \mathrm{~min}$. The intensity of the exercise sessions was set at heart rate (HR) corresponding to the interval between the ventilator anaerobic threshold (VAT) and respiratory compensation point (RCP). Shortly after each aerobic session, the patient performed resistance training, which was comprised seven exercises 
per session, including leg press, bench press, leg extension, pulley, leg flexion, seated row and abdominal exercises. The patient performed three sets of 12 repetitions, with $1 \mathrm{~min}$ rest between sets. Stretching exercises were performed after the resistance training. Training intensity was adjusted according to a gradual increase in strength, such that the patient was able to perform no more than 12 repetition maximum (RM). At least two physical education professional supervised and monitored all sessions. The patient was advised to report any adverse events throughout the exercise training program.

Before and after the exercise training program, the patient completed a maximal graded treadmill cardiopulmonary exercise test to determine peak oxygen uptake (peak $\mathrm{VO}_{2}$ ). Additionally, muscle strength tests (assessed by one-RM and handgrip test), functional performance (assessed by Timed Up and Go [TUG] and Timed-Stands Test [TST]), were evaluated as described elsewhere [9] [10]. Fat-free mass, fat mass and body fat were measured using dual X-ray absorptiometry (DXA).

Data on aerobic capacity and muscle strength at baseline and after 12-week of exercise intervention are given in Table 1. After the 12-week exercise training program, the patient exhibited a substantial increase in aerobic capacity, demonstrated by increased time to ventilatory anaerobic threshold, time torespiratory compensation point and time to exhaustion, with no changes in absolute $(\mathrm{L} / \mathrm{min})$ or relative $(\mathrm{mL} / \mathrm{kg} / \mathrm{min})$ peak $\mathrm{VO}_{2}$. Moreover, increases in peak heart rate peak and respiratory exchange rate were observed. In addition, muscle strength was increased on the leg press and bench press exercises and improvement was seen in the TUG test, suggesting improved muscle function.

Body composition data are presented in Figure 1. The patient had a significant decrease in body fat and fat mass as well as an increase in fat-free mass, suggesting improved body composition after the 12-weeks exercise training program. The patient signed the consent form and consents with the publishing of this case report.

\section{Discussion}

This is the first study to evaluate the safety and benefits of exercise training in a patient with RP. The present study showed that a 12-week exercise training was able to increase aerobic capacity, as well as upper- and lower-limb muscle strength and functional capacity. In addition, a decrease in body fat and fat mass and an increase in fat-free mass were also observed. Notably, there was no disease relapse or adverse events during the exercise training program.

Patients with RP have recurrent inflammation in proteogly can-rich structures and cartilaginous tissues, which can lead to clinical manifestations, such as respiratory tract chondritis, airway collapse, valvar cardiac involvement, inflammation of peripheral joints and non-erosive polyarthritis [1] [2] [3] [4] [5]. All these outcomes can contribute to impairment in aerobic capacity and decrease in muscle strength and function in patients with RP. 
Table 1. Aerobic capacity, muscle strength and functional capacity in the patient at baseline and after 12-weeks exercise training program.

\begin{tabular}{|c|c|c|c|}
\hline & At baseline & After 12 weeks & Variation (\%) \\
\hline \multicolumn{4}{|l|}{ Aerobic capacity } \\
\hline Peak $\mathrm{VO}_{2}(\mathrm{~L} / \mathrm{min})$ & 1.2 & 1.2 & 0.0 \\
\hline Peak $\mathrm{VO}_{2}(\mathrm{~mL} / \mathrm{kg} / \mathrm{min})$ & 21.0 & 21.4 & +1.9 \\
\hline Peak Heart rate (bpm) & 144 & 176 & +22.2 \\
\hline PeakRER & 0.9 & 1.3 & +44.4 \\
\hline Time to VAT (min) & 2.3 & 6.0 & +160.9 \\
\hline Time to RCP (min) & 4.3 & 10.0 & +132.6 \\
\hline Time to exhaustion (min) & 6.0 & 12.0 & +100.0 \\
\hline \multicolumn{4}{|c|}{ Strength and functional capacity } \\
\hline Legpress 1RM (kg) & 100.0 & 120.0 & +20.0 \\
\hline Benchpress 1RM (kg) & 22.0 & 24.0 & +9.1 \\
\hline Handgrip (kg) & 22.0 & 21.5 & -2.3 \\
\hline TST (reps) & 15 & 17 & +13.3 \\
\hline TUG (s) & 6.9 & 6.5 & -6.4 \\
\hline
\end{tabular}

RER: respiratory exchange rate; RCP: respiratory compensation point; RM: repetition maximum; TST: timed-stands test; TUG: timed-up-and-go. VAT: ventilatory anaerobic threshold; Peak $\mathrm{VO}_{2}$ : peak oxygen uptake.
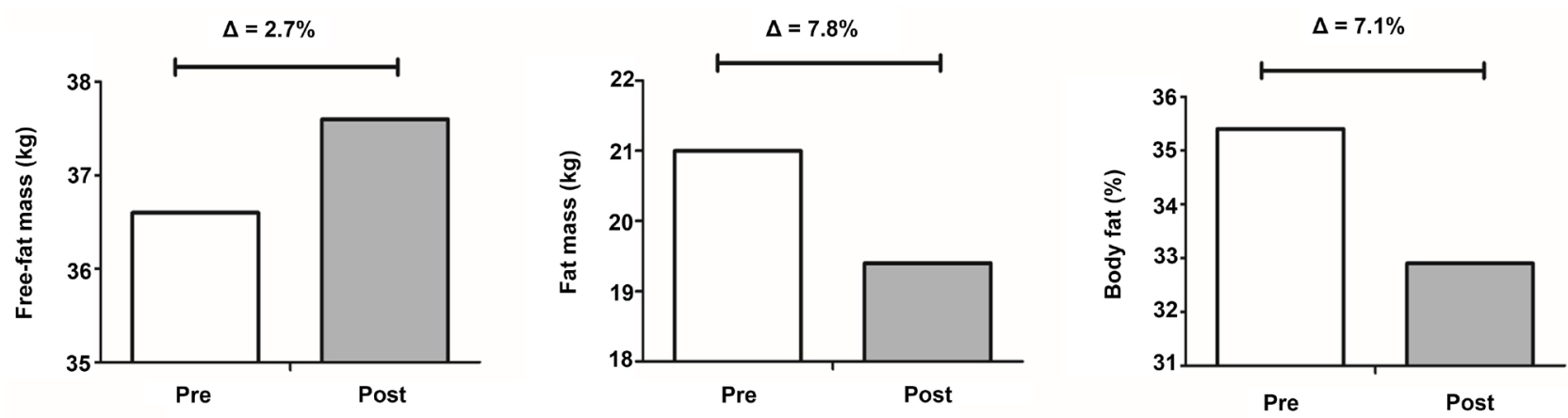

Figure 1. Body composition in the patient before and after 12-week exercise training program.

Impairmentsin aerobic capacity, muscle strength and function in autoimmune rheumatic disease have been associated with disease activity, impaired quality of life and increased cardiovascular risk in patients with autoimmune rheumatic diseases [11] [12]. In the present study, a 12-week exercise training program was shown to be effective for improving aerobic capacity and increasing muscle strength and function (i.e. TUG) in the RP patient, with no related adverse events reported, suggesting a potential therapeutic effect of exercise training in this disease.

There have been concerns that exercise training may increase inflammation and exacerbate clinical symptoms in autoimmune rheumatic disease [13]. However, sedentary behavior, which leads to an increase in adipose tissue, has been 
strongly associated with worsening inflammation [14] [15]. Notably, recent studies have demonstrated that exercise has a potential anti-inflammatory effect in inflammatory diseases [9] [10]. One mechanism that contributes to the anti-inflammatory effects of exercise training is a decrease in adipose tissue [14]. In the present study, substantial decreases in body fat and fat mass, besides an increase in fat-free mass, were observed. These results suggest that exercise training can improve body composition and possibly help attenuate inflammatory markers in RP. Inflammatory markers were not evaluated in this case study and therefore further studies involving a larger number of patients are necessary to confirm this assumption.

\section{Conclusion}

In conclusion, the present case report suggests that a chronic exercise training program increased aerobic capacity, muscle strength and functional capacity in the patient. Additionally, a decrease in body fat and fat mass, and an increase in fat-free mass, suggests that exercise training can improve body composition in this disease. No adverse events were reported during the exercise training program, indicating that the exercise training was safe in this case study. However, studies assessing inflammatory markers, safety and the exercise training program in the disease are necessary to confirm these findings.

\section{Conflicts of Interest}

All authors declare no conflict of interest.

\section{Fund}

This work was supported by Fundação de Amparo à Pesquisa do Estado de São Paulo (FAPESP) [\#2016/19771-5 to D.S.O, \#2016/23574-0 to R.G.M., \#2017/ 06794-0 to L.A.P]; Federico Foundation, Faculdade de Medicina da USP and Fundação Faculdade de Medicina to S.K.S.

\section{References}

[1] Trentham, D.E. and Le, C.H. (1998) Relapsing Polychondritis. Annals of Internal Medicine, 129, 114-122. https://doi.org/10.7326/0003-4819-129-2-199807150-00011

[2] McAdam, L.P., O’Hanlan, M.A., Bluestone, R. and Pearson, C.M. (1976) Relapsing Polychondritis: Prospective Study of 23 Patients and a Review of the Literature. Medicine (Baltimore), 55, 193-215. https://doi.org/10.1097/00005792-197605000-00001

[3] Pallo, P.A.O., Levy-Neto, M., Pereira, R.M.R. and Shinjo, S.K. (2017) Relapsing Polychondritis: Prevalence of Cardiovascular Diseases and Its Risk Factors, and General Disease Features According to Gender. Revista Brasileira de Reumatologia Engl Ed, 57, 338-345. https://doi.org/10.1016/j.rbr.2017.01.002

[4] Schumacher, S. and Pieringer, H. (2017) Relapsing Polychondritis: A Chameleon among Orphan Diseases. Wiener Medizinische Wochenschrift, 167, 227-233. https://doi.org/10.1007/s10354-017-0559-1 
[5] Michet Jr., C.J., McKenna, C.H., Luthra, H.S. and O'Fallon, W.M. (1986) Relapsing Polychondritis. Survival and Predictive Role of Early Disease Manifestations. Annals of Internal Medicine, 104, 74-78. https://doi.org/10.7326/0003-4819-104-1-74

[6] Perandini, L.A., de Sá-Pinto, A.L., Roschel, H., Benatti, F.B., Lima, F.R., Bonfá, E. and Gualano, B. (2012) Exercise as a Therapeutic Tool to Counteract Inflammation and Clinical Symptoms in Autoimmune Rheumatic Diseases. Autoimmunity Reviews, 12, 218-224. https://doi.org/10.1016/j.autrev.2012.06.007

[7] Benatti, F.B. and Pedersen, B.K. (2015) Exercise as an Anti-Inflammatory Therapy for Rheumatic Diseases-Myokine Regulation. Nature Reviews Rheumatology, 11, 86-97. https://doi.org/10.1038/nrrheum.2014.193

[8] Stavropoulos-Kalinoglou, A., Metsios, G.S., Veldhuijzen van Zanten, J.J., Nightingale, P., Kitas, G.D. and Koutedakis, Y. (2013) Individualised Aerobic and Resistance Exercise Training Improves Cardiorespiratory Fitness and Reduces Cardiovascular Risk in Patients with Rheumatoid Arthritis. Annals of the Rheumatic Diseases, 72, 1819-1825. https://doi.org/10.1136/annrheumdis-2012-202075

[9] Perandini, L.A., Oliveira, D.S., Mello, S.B., Camara, N.O., Benatti, F.B., Lima, F.R., Borba, E., Bonfa, E., Sá-Pinto, A.L., Roschel, H. and Gualano, B. (2014) Exercise Training Can Attenuate the Inflammatory Milieu in Women with Systemic Lupus Erythematosus. Journal of Applied Physiology, 117, 639-647.

https://doi.org/10.1152/japplphysiol.00486.2014

[10] Oliveira, D.S., Shinjo, S.K., Silva, M.G., de Sá-Pinto, A.L., Lima, F.R., Roschel, H., Mello, S.B.V., Costa-Hong, V., Irigoyen, M.C.C., Pereira, R.M. and Gualano, B. (2017) Exercise in Takayasu Arteritis: Effects on Inflammatory and Angiogenic Factors and Disease-Related Symptoms. Arthritis Care \& Research (Hoboken), 69, 892-902. https://doi.org/10.1002/acr.23011

[11] Khoja, S.S., Almeida, G.J., Chester Wasko, M., Terhorst, L. and Piva, S.R. (2016) Association of Light-Intensity Physical Activity with Lower Cardiovascular Disease Risk Burden in Rheumatoid Arthritis. Arthritis Care \& Research (Hoboken), 68, 424-431. https://doi.org/10.1002/acr.22711

[12] Poulsen, K.B., Alexanderson, H., Dalgard, C., Jacobsen, S., Weile, L. and Diederichsen, L.P. (2017) Quality of Life Correlates with Muscle Strength in Patients with Dermato- or Polymyositis. Clinical Rheumatology, 36, 2289-2295. https://doi.org/10.1007/s10067-017-3706-6

[13] Partridge, R.E. and Duthie, J.J. (1963) Controlled Trial of the Effect of Complete Immobilization of the Joints in Rheumatoid Arthritis. Annals of the Rheumatic Diseases, 22, 91-99. https://doi.org/10.1136/ard.22.2.91

[14] Allison, M.A., Jensky, N.E., Marshall, S.J., Bertoni, A.G. and Cushman, M. (2012) Sedentary Behavior and Adiposity-Associated Inflammation: The Multi-Ethnic Study of Atherosclerosis. American Journal of Preventive Medicine, 42, 8-13. https://doi.org/10.1016/j.amepre.2011.09.023

[15] Henson, J., Yates, T., Edwardson, C.L., Khunti, K., Talbot, D., Gray, L.J., Leigh, T.M., Carter, P. and Davies, M.J. (2013) Sedentary Time and Markers of Chronic Low-Grade Inflammation in a High Risk Population. PLoS One, 8, e78350. https://doi.org/10.1371/journal.pone.0078350 